\title{
A multimodal corpus for technology-enhanced learning of violin playing
}

\author{
Gualtiero Volpe \\ DIBRIS - University of Genova \\ Genova, Italy \\ gualtiero.volpe@unige.it \\ Simone Ghisio \\ DIBRIS - University of Genova \\ Genova, Italy \\ simoneghisio@gmail.com \\ Stefano Piana \\ DIBRIS - University of Genova \\ Genova, Italy \\ stefano.piana@dist.unige.it
}

\author{
Ksenia Kolykhalova \\ DIBRIS - University of Genova \\ Genova, Italy \\ ksenia.kolykhalova@dibris.unige.it \\ George Waddell \\ Royal College of Music \\ London, United Kingdom \\ george.waddell@rcm.ac.uk \\ Corrado Canepa \\ DIBRIS - University of Genova \\ Genova, Italy \\ corrado@infomus.org
}

\author{
Erica Volta \\ DIBRIS - University of Genova \\ Genova, Italy \\ erica.volta@edu.unige.it \\ Paolo Alborno \\ DIBRIS - University of Genova \\ Genova, Italy \\ paoloalborno@gmail.com \\ Rafael Ramirez-Melendez \\ Universitat Pompeu Fabra \\ Barcelona, Spain \\ rafael.ramirez@upf.edu
}

\begin{abstract}
Learning to play a musical instrument is a difficult task, mostly based on the master-apprentice model. Technologies are rarely employed and are usually restricted to audio and video recording and playback. Nevertheless, multimodal interactive systems can complement actual learning and teaching practice, by offering students guidance during self-study and by helping teachers and students to focus on details that would be otherwise difficult to appreciate from usual audiovisual recordings. This paper introduces a multimodal corpus consisting of the recordings of expert models of success, provided by four professional violin performers. The corpus is publicly available on the repoVizz platform, and includes synchronized audio, video, motion capture, and physiological (EMG) data. It represents the reference archive for the EUH2020-ICT Project TELMI, an international research project investigating how we learn musical instruments from a pedagogical and scientific perspective and how to develop new interactive, assistive, self-learning, augmented-feedback, and social-aware systems to support musical instrument learning and teaching.
\end{abstract}

Permission to make digital or hard copies of part or all of this work for personal or classroom use is granted without fee provided that copies are not made or distributed for profit or commercial advantage and that copies bear this notice and the full citation on the first page. Copyrights for thirdparty components of this work must be honored. For all other uses, contact the owner/author(s).

CHItaly '17, September 18-20, 2017, Cagliari, Italy

(C) 2017 Copyright held by the owner/author(s).

ACM ISBN 978-1-4503-5237-6/17/09.

https://doi.org/10.1145/3125571.3125588

\section{CCS CONCEPTS}

- Applied computing $\rightarrow$ Sound and music computing; Computer-assisted instruction; Interactive learning environments;

\section{KEYWORDS}

Multimodal corpora, multimodal interactive systems, technology-enhanced learning, music performance.

\section{ACM Reference format:}

Gualtiero Volpe, Ksenia Kolykhalova, Erica Volta, Simone Ghisio, George Waddell, Paolo Alborno, Stefano Piana, Corrado Canepa, and Rafael Ramirez-Melendez. 2017. A multimodal corpus for technologyenhanced learning of violin playing. In Proceedings of CHItaly '17, Cagliari, Italy, September 18-20, 2017, 5 pages.

https://doi.org/10.1145/3125571.3125588

\section{INTRODUCTION}

Learning to play a musical instrument is mostly based on the master-apprentice model. In this difficult task, student's interaction with teachers is often restricted to short and punctual contacts followed by long periods of self-study resulting in high abandonment rates. Technologies are rarely employed and are usually restricted to audio and video recording and playback. The EU-H2020-ICT TELMI Project ${ }^{1}$ takes the violin as a case-study to investigate how we learn musical instruments from a pedagogical and scientific perspective and to develop new interactive, assistive, self-learning, augmentedfeedback, and social-aware systems to support musical instrument learning and teaching.

In this framework, this paper introduces the recordings of expert models of success, provided by four professional

${ }^{1}$ http://telmi.upf.edu/ 
violin performers and teachers between December 2016 and January 2017. Recordings are collected in a publicly available reference archive and consist of synchronized audio, video, motion capture, and physiological (EMG) data. The multimodal corpus is currently used for research, technolog$\mathrm{ical}$, and educational purposes, including developing feature computation and analysis techniques, modeling and automatically measuring the technical quality of a violin performance, comparing students with masters to provide ways of improving students' technique, designing feedback for students, and providing students with reference examples of performances. The final goal is to develop a multimodal interactive system for technology-enhanced learning of violin playing, capable of analyzing violin performances both in real-time and off-line and of providing teachers and students with interactive feedback. The system can complement actual learning and teaching practice, by offering guidance during self-study and by helping teachers and students to focus on details that would be otherwise difficult to appreciate from usual audiovisual recordings.

The paper is organized as follows: the next section introduces some related work; then, the collected data corpus is described; finally, future developments are sketched.

\section{RELATED WORK}

Several online resources are available on the Internet including data corpora concerning music in general and music performance in particular. Resources include repositories of audio samples (e.g., Freesound ${ }^{2}$ ), repositories of music pieces such as for example Free Music Archive ${ }^{3}$, Jamendo Music $^{4}$, and the medleyDB dataset [2], and repositories created on-purpose for specific research challenges, e.g., the datasets used in the tasks of the Music Information Retrieval Evaluation eXchange (MIREX) ${ }^{5}$ and in the Emotion in $\mathrm{Mu}-$ sic task in MediaEval [7][1]. These corpora, however, only contain audio data and are therefore unsuitable for a deeper analysis of music performance, taking also into account e.g., the performer's posture and movement. To this aim, corpora including data from multiple sources e.g., motion capture, audio, and video were collected. The Bowstroke Database [9], for example, contains calibrated gesture and audio data corresponding to various violin bowstrokes. A subset of the database includes video and motion capture data as well. Other relevant examples are the ENSTDrums dataset [4], a rich collection of annotated audiovisual recordings of drum performances for automatic drum transcription and processing, the motion capture database of music performances

\footnotetext{
${ }^{2} \mathrm{http}: / /$ freesound.org/

${ }^{3} \mathrm{http}: / /$ freemusicarchive.org/

${ }^{4} \mathrm{http}: / /$ jamendo.com/

${ }^{5} \mathrm{http} / / /$ www.music-ir.org/mirex/wiki/MIREX_HOME
}

collected at University of Oslo ${ }^{6}$, the corpora collected in the framework of the Interpersonal Entrainment in Music Performance (IEMP) project at Durham University ${ }^{7}$, and the Quartet Dataset [5], a multimodal music performance dataset focusing on the string quartet ensemble originally aimed at studying the interdependence between the members of the quartet and at modeling ensemble expressive performance. The latter dataset is made available through repoVizz ${ }^{8}$, an online platform for open access to collections of time-aligned multimodal datastreams, containing several archives of music performance data.

With respect to existing corpora, the one described in this paper has the special purpose to be recorded in view of the development of a multimodal interactive system for technology-enhanced learning of violin playing. As such, it is not oriented to a specific research task (e.g., emotion recogition) or to the performance of particular music pieces. Rather, it is organized in a structured collection of exercises, following a precise learning path. Moreover, it collects data for several rich sources, including motion capture, audio, video, and physiological sensors.

\section{DATA CORPUS}

\section{Participants}

Four internationally renowned professional violin players were recruited for recording the corpus. Madeleine Mitchell is Professor at the Royal College of Music in London. She is a violin soloist, chamber musician, dedicated teacher, and artistic director. She has performed in some 50 countries and made many recordings and broadcast. John Haspel Gilbert is Professor of Violin at the Texas Tech University School of Music in Lubbock, Texas. He is the recipient of the 2015 TexASTA Phyllis Young Outstanding Studio Teacher Award, the TTU President's Award for Excellence in Teaching, and served as concertmaster of the Lubbock Symphony Orchestra for 13 seasons. Eulalie Charland is an instrumental teacher at the University of Chichester and a chamber music coach at the Guildhall School of Music and Drama. She is an accomplished violinist, with an international performance and teaching profile. Berent Korfker is Professor at the Royal College of Music in London and the Yehudi Menuhin School in Surrey. He has performed concertos with major orchestras worldwide and has made solo recital broadcasts in the USA, France, and the Netherlands.

\section{Material}

Material consisted of 41 exercises concerning:

\footnotetext{
${ }^{6} \mathrm{http} / /$ www.uio.no/english/research/groups/fourms/downloads/ motion-capture/

${ }^{7}$ https://musicscience.net/projects/iemp/

${ }^{8}$ https://repovizz.upf.edu/repo/Home
} 
A multimodal corpus for technology-enhanced learning of violin playing CHItaly'17, September 18-20, 2017, Cagliari, Italy

- Handling the instrument: posture, holding the violin, holding the bow;

- Techniques of the right hand: bow control, bow direction, bow changes, bow tilt, bow speed, bow weight, bow contact point, string crossing;

- Techniques of the left hand: articulation, intonation, shifts, double stops, vibrato, trill.

- Articulation: détaché, legato, martelé, pizzicato, sautillé, spiccato, staccato, ricochet.

Exercises were selected under the guidance of professional violinist Madeleine Mitchell - who also participated in the recordings - and supported through consultation with performing, teaching, and student violinists. The exercises take three forms: (1) those sourced from standard published catalogs of exercises, including those of Schradieck, Ševčík, and Kreutzer; (2) those sourced or adapted from the Associated Board of the Royal Schools of Music (ABRSM) examination syllabus; and (3) customized exercises developed by Madeleine Mitchell to address specific techniques with a particular focus on the capabilities offered by non-notated feedback (e.g., the bowing exercises). The use of both custom and pre-existing exercises was deliberate. The bespoke pieces are intended to capitalize on the unique possibilities offered by audio, video, and motion-capture feedback, while the published sources draw on centuries of pedagogical insight and tradition to give students and teachers a common frame of reference.

\section{Set-up}

Recordings consisted of motion capture data (performer, violin, and bow), instrument and ambient audio, video (frontal and lateral view), and data from physiological sensors (EMG as captured by Myo sensors ${ }^{9}$ ). Equipment included:

- A 13-cameras Qualysis motion capture system;

- Two video cameras JVC GY-HD251 (720p, 50 fps);

- One Kinect v.2, capturing both live video and depthmap;

- One pick-up Fishman PRO-V20-0VI connected via radio (AKG UHF wireless system PT40-SR40) mounted on the violin;

- Two Neumann KM184 microphones for ambient audio;

- Two Myo sensor for EMG data.

Figure 1 shows a top and a frontal view of the environment for the recordings and displays were equipment was located. Recordings were carried out at the Casa Paganini-InfoMus research center of DIBRIS-University of Genova, Italy.

The violin was endowed with 6 small lightweight reference reflective markers and with a pick-up microphone. A $6 \mathrm{DOF}$ rigid body was defined to track the violin in the local systems of coordinates. Virtual markers were used for

\footnotetext{
${ }^{9}$ http://www.myo.com/
}
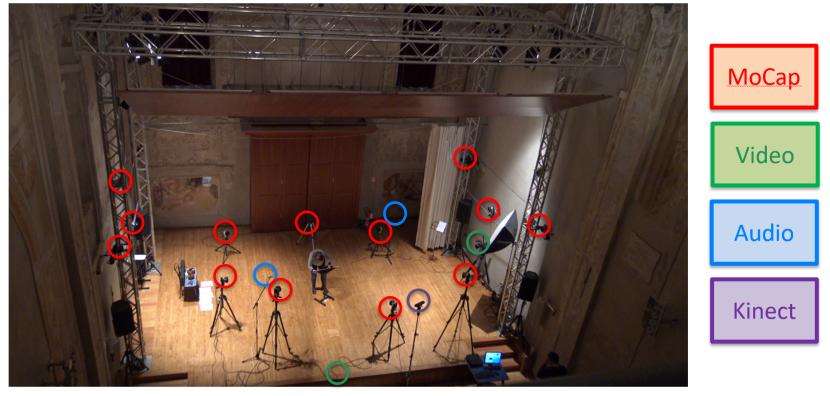

(a) Top view.
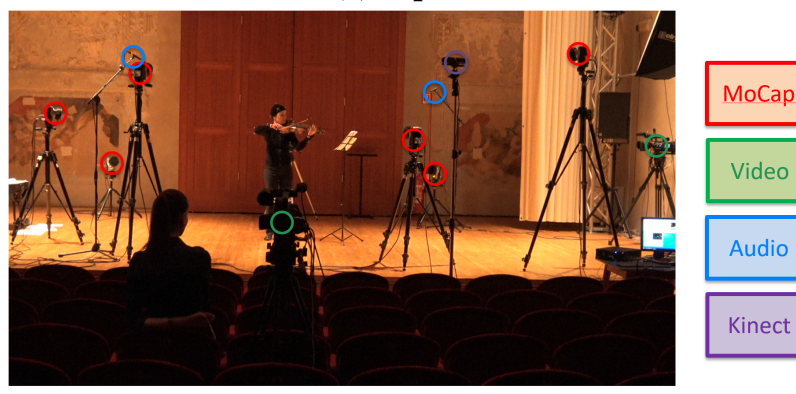

(b) Frontal view.

Figure 1: Top (a) and frontal (b) view of the set-up. Colors show the locations where the video cameras of the motion capture system, the video cameras for video recordings, the microphones for ambient audio recordings, and the Kinect sensor were positioned.

the violin strings. These are markers that are placed at each string-end during the calibration phase. Then markers are removed for the performance, as they would be very intrusive. During the actual tracking, virtual markers are reconstructed from the reference markers. After the first recording session with Madeleine Mitchell, the same violin was used for the next three performers. This choice had a twofold benefit: (i) it reduced the variability introduced by using different music instruments (i.e., it reduced the number of variables to take into account), and (ii) it enabled speeding-up the recordings, since the violin could be prepared in advance by putting motion capture markers and the pick-up microphone in appropriate positions on its surface. Some time was needed for the players to get acquainted with the new instrument. The players, however, are all professionals. Figure 2 shows where the reflective markers, the virtual markers, and the pick-up microphone were positioned on the violin.

The bow was also endowed with lightweight reflective markers and a 6DOF rigid body was defined to track it (see figure 3) The same bow (prepared in advance) was used with John H. Gilbert and with Eulalie Charland, whereas Berent Korfker preferred to use his own bow. Both the violin and 


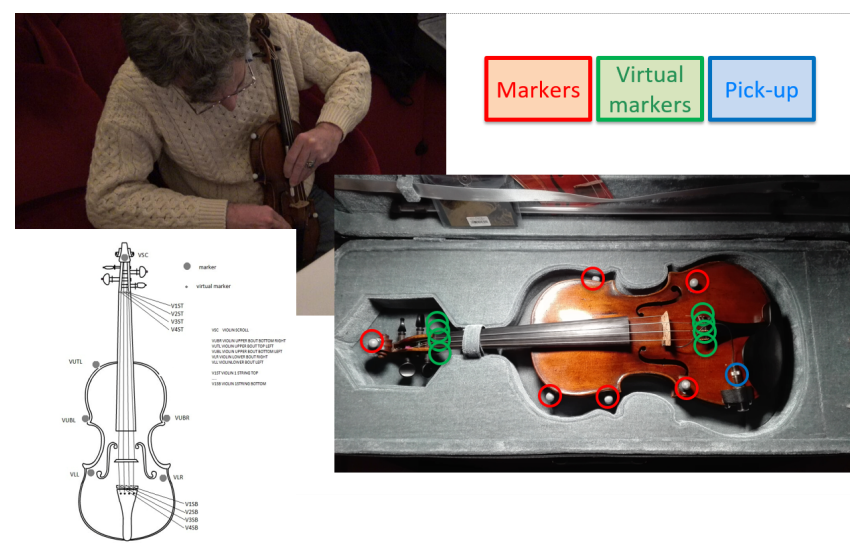

Figure 2: Preparation of the violin. Reflective markers and a pick up microphone are placed on the surface of the violin. Moreover, virtual markers are used.

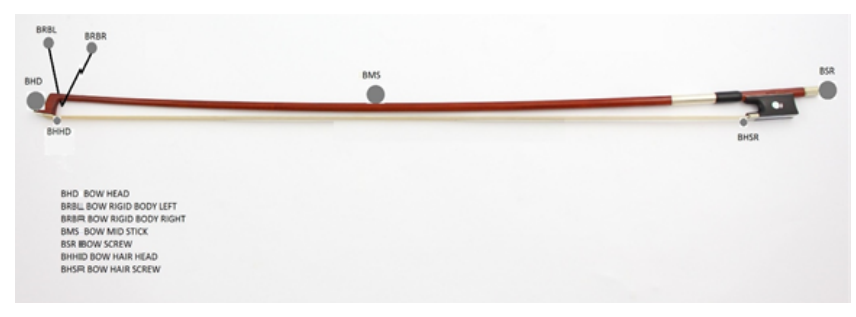

Figure 3: Positions of the reflective markers on the bow.

the bow were kindly provided by Alberto Giordano an internationally renowned luthier, conservator of the Cannone, the violin owned by Niccolò Paganini.

Markers on the body of the player were placed as follows: right and left front head, right and left back head, head top, C7 spine, spine fifth thoracic vertebra, spine tenth thoracic vertebra, left and right shoulder, left and right scapula, left and right knee (inner and outer side), right and left pelvic bone back, sacrum bone, right and left toe, right and left elbow (inner and outer side), right and left wrist (inner and outer side), right and left palm index finger, right and left palm pinky finger. A motion capture suit of appropriate size was used. Moreover, two Myo bracelet were placed on the left and right forearms.

The EyesWeb XMI platform ${ }^{10}$ [3][8] was used for synchronized data recording and play back. Synchronization is based on SMPTE time-stamps. Kinect and Myo data were synchronized by EyesWeb XMI using proprietary time-stamps.

\footnotetext{
${ }^{10}$ www.infomus.org
}

\section{Procedure}

A document was prepared in advance and shared with each of the participants to explain the requirements for the recording, e.g., the need to wear a motion capture suit and the physiological sensors as well as the need to play a violin prepared with markers. Upon arrival at the location of the recordings, after initial welcoming and further explanation of the purpose and procedure of the recordings, each participant was dressed with a motion capture suit of appropriate size and was given time to get used to play in the required conditions. At the same time or immediately before starting the recording session, the motion capture system was calibrated and a recording test was performed to check whether all the required equipment was working properly. During the recording session, the player was free to play the exercises in the order $\mathrm{s} /$ he preferred. The player could replay each exercise how many times s/he wanted, until s/he was satisfied with the performance. Players were also given the opportunity to check the recordings after the session and to record again the exercises they were not satisfied with. The players were informed of the purpose of the recording and signed a consent form.

\section{Results}

The recorded material was post-processed and uploaded in the repoVizz repository, where it is publicly available. Figure 4 shows a sample item of the corpus in the repoVizz web interface. EyesWeb Mobile interfaces were also developed for preview and play back of downloaded data (see Figure 5).

Data is currently being analyzed to extract audio and movement features. Concerning the latter, computed features include kinematics (velocity, acceleration, jerk, curvature of relevant trajectories such as hands and head, rangles between bones, kinetic energy) and higher level features, such as openness, lightness, sway, tension, suddenness, coordination. An ongoing work consists, for example, in analyzing regularity of movement signals and movement coordination (intended as intra-personal synchronization). In this framework, figure 6 shows an application of Recurrence Quantification Analysis [6] to the kinetic energy of the right wrist. Recurrence rates of signals captured from players having a different level of skill can be compared, e.g., as a contribute for assessing the technical quality of the music performance.

\section{CONCLUSION AND FUTURE WORK}

This paper presented a multimodal corpus of violin performances. These consist of 41 exercises performed by 4 internationally renowned professional violin players. In the near future, the corpus is going to be extended in two major directions. First, further recordings exploiting the Qualysis motion capture system will be carried out. These recordings 
A multimodal corpus for technology-enhanced learning of violin playing CHItaly'17, September 18-20, 2017, Cagliari, Italy

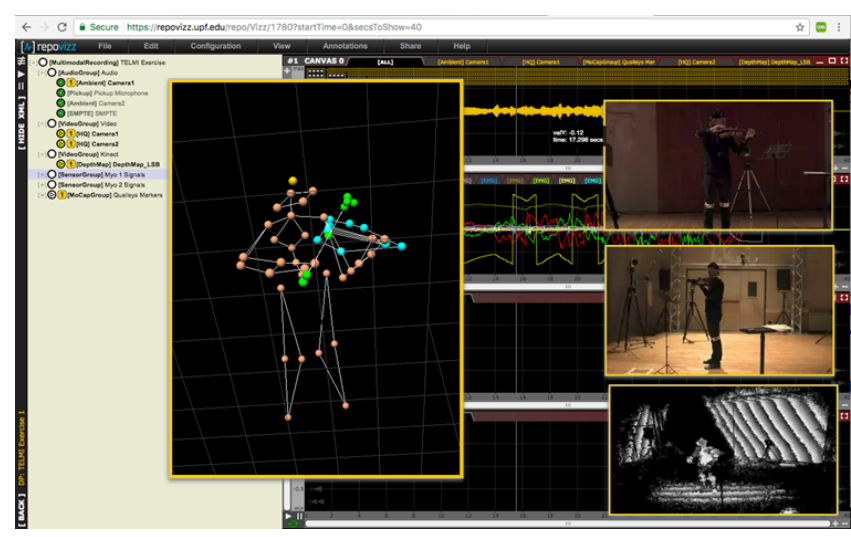

Figure 4: A sample item of the corpus in the repoVizz web interface.

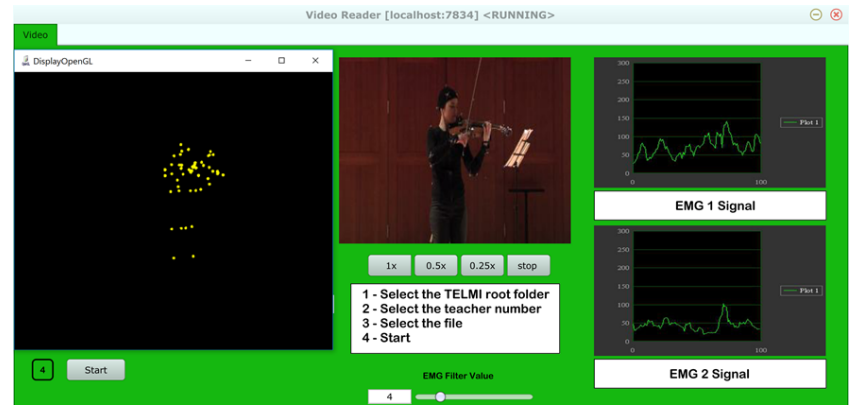

Figure 5: An EyesWeb Mobile interface for preview and play back of the data.

will be performed by other professional players as well as highly and medium skilled amateur players and conservatory students. The purpose is to quantify and model the technical quality of the recorded performances, and to compare technical quality between performances by experts and performances by amateurs and students having different levels of skill. Secondly, further recordings will be made by using low-cost technologies (e.g., Kinect and/or other low-cost sensor systems). The purpose is to port technologies to more affordable devices, available to both students and schools.

\section{ACKNOWLEDGMENTS}

We thank violinists Madeleine Mitchell, John H. Gilbert, Eulalie Charland, and Berent Korfker for participating in the recordings. We thank luthier Alberto Giordano for providing and preparing the violin and the bow. We thank the staff of the Casa Paganini - InfoMus research center and the partners in the TELMI project for their help. This work is partially supported by the EU-H2020-ICT Project TELMI. TELMI has received funding from the European Union's Horizon 2020 Research and Innovation Programme under Grant Agreement No. 688269.

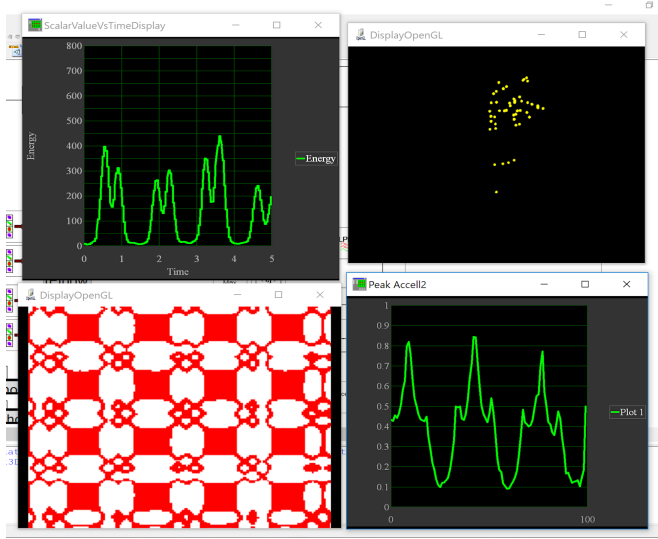

Figure 6: Recurrence Quantification Analysis applied to kinetic energy of the right wrist. In the bottom, recurrence plot and probabilities of recurrence of the signal in the top.

\section{REFERENCES}

[1] Anna Aljanaki, Yi-Hsuan Yang, and Mohammad Soleymani. 2017. Developing a benchmark for emotional analysis of music. PLOS ONE 12, 3 (03 2017), 1-22.

[2] Rachel Bittner, Justin Salamon, Mike Tierney, Matthias Mauch, Chris Cannam, and Juan Bello. 2014. MedleyDB: A multitrack dataset for annotation-intensive MIR research. In Proceedings of the 15th International Society for Music Information Retrieval Conference (ISMIR 2014).

[3] Antonio Camurri, Shuji Hashimoto, Matteo Ricchetti, Andrea Ricci, Kenji Suzuki, Riccardo Trocca, and Gualtiero Volpe. 2000. EyesWeb: Toward Gesture and Affect Recognition in Interactive Dance and Music Systems. Computer Music Journal 24, 1 (April 2000), 57-69.

[4] Olivier Gillet and Gaël Richard. 2006. Enst-drums: an extensive audiovisual database for drum signals processing. In Proceedings of the 7th International Symposium on Music Information Retrieval (ISMIR 2006).

[5] Esteban Maestre, Panagiotis Papiotis, Marco Marchini, Quim Llimona, Oscar Mayor, Alfonso Pérez, and Marcelo M. Wanderley. 2017. Enriched Multimodal Representations of Music Performances: Online Access and Visualization. IEEE MultiMedia 24, 1 (Jan 2017), 24-34.

[6] Norbert Marwan, M. Carmen Romano, Marco Thiel, and Jürgen Kurths. 2007. Recurrence plots for the analysis of complex systems. Physics Reports 438 (2007), 237-329.

[7] Mohammad Soleymani, Anna Aljanaki, Yi-Hsuan Yang, Michael N. Caro, Florian Eyben, Konstantin Markov, Björn W. Schuller, Remco Veltkamp, Felix Weninger, and Frans Wiering. 2014. Emotional Analysis of Music: A Comparison of Methods. In Proceedings of the 22nd ACM International Conference on Multimedia (MM '14). ACM, New York, NY, USA, 1161-1164.

[8] Gualtiero Volpe, Paolo Alborno, Antonio Camurri, Paolo Coletta, Simone Ghisio, Maurizio Mancini, Radoslaw Niewiadomski, and Stefano Piana. 2016. Designing Multimodal Interactive Systems using EyesWeb XMI. In Proceedings of the First International Workshop on Smart Ecosystems cReation by Visual dEsign co-located with the International Working Conference on Advanced Visual Interfaces (AVI 2016), Bari, Italy, June 07, 2016. 49-56.

[9] Diana Young and Anagha Deshmane. 2007. Bowstroke Database: A Web-accessible Archive of Violin Bowing Data. In Proceedings of the 7th International Conference on New Interfaces for Musical Expression (NIME '07). ACM, New York, NY, USA, 352-357. 